\title{
Genetic variation of the platelet- surface integrin GPIIb-Illa (PIA1/A2-SNP) shows a high association with Type 2 diabetes mellitus
}

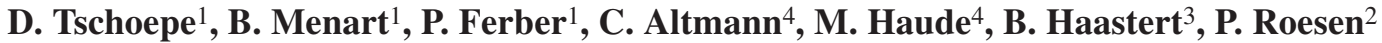 \\ ${ }^{1}$ German Diabetes Clinic, German Diabetes Research Institute at the Heinrich Heine University of Duesseldorf, Duesseldorf, Germany \\ 2 Department of Pathobiochemistry, German Diabetes Research Institute at the Heinrich Heine University of Duesseldorf, Germany \\ ${ }^{3}$ Department of Epidemiology and Biostatistics, German Diabetes Research Institute at the Heinrich Heine University of Duesseldorf, \\ Germany \\ ${ }^{4}$ Department of Cardiology, University Hospital of Essen, Germany
}

\section{Abstract}

Aims/hypothesis. The gene encoding the $\beta_{3}$-subunit (GPIIIa) of the platelet $\alpha_{2} \beta_{3}$-integrin (fibrinogen receptor) shows a polymorphism PlA1/A2 with the $A 2$ allele putatively associated with an increased risk of acute ischaemic events. This study investigated whether Type 2 diabetes as a particular macrovascular risk factor associates with the thrombogenic PIA2 genotype.

Methods. The PlA genotype was determined in 112 consecutive Type 2 diabetic patients additionally classified according to the presence of macrovascular disease. Forty-four non-diabetic patients with angiografically documented cardiovascular disease (CAD/ AMI) and a further 59 non-diabetic subjects with no angiografical signs of CAD were investigated as genomic background control $(n=103)$. PIA-genotyping was carried out by standard restriction fragment length analysis (RFLA) of PCR amplified lymphocyte template DNA.

Results. The overall allelic PlA2- prevalence accounted to $34.8 \%$ (39/112) in diabetic patients as compared to $14.6 \%(15 / 103)$ in non-diabetic patients [OR 3.1 (1.6-6.1), $p<0.01]$.This odds ratio increased to 7.0
(2.5-19.7), $(p<0.01)$ in subjects free of criteria of macrovascular disease. In non-diabetic control subjects without CAD there was an allelic PIA2 frequency of $10.2 \%(6 / 59)$ as compared to $20.5 \%(9 / 44)$ in patients with CAD and a history of AMI being less than either diabetes subgroup. The PIA2 prevalence in the subgroup of diabetes patients with macrovascular complications did not differ from the respective value in patients without macrovascular disease. [29.0\% (20/69) vs. $44.2 \%$ (19/43)].

Conclusion/interpretation. This study confirms a trendwise association of PlA2 with severe coronary artery disease, but rather suggests an even stronger, highly significant association with the metabolic condition of Type 2 diabetes mellitus. This justifies the speculation that pathways dependent on the platelet $\alpha_{2} \beta_{3}$ integrin physiology could be implicated in the pathogenesis of Type 2 diabetes which lends further support to the "common soil" hypothesis of diabetes and vascular disease. [Diabetologia (2003) 46:984-989]

Keywords Fibrinogen receptor PIA1/PIA2 polymorphism, platelets, Type 2 diabetes mellitus, vascular complications.
Received: 23 October 2002 / Revised: 27 March 2003

Published online: 21 June 2003

(C) Springer-Verlag 2003

Corresponding author: Dr. D. Tschoepe, German Diabetes Clinic, German Diabetes Research Institute at the Heinrich Heine University of Duesseldorf, Auf'm Hennekamp 65, 40225 Duesseldorf, Germany

E-mail: dtschoepe@hdz.nrw.de

Abbreviations: AMI, acute myocardial infarction; CAD, coronary artery disease; GPIIIa, $\beta_{3}$ subunit of the platelet surface $\alpha_{2} \beta_{3}$-integrin (GPIIb-IIIa, fibrinogen-receptor); PIA, platelet antigen; RGD, aminoacidsequence Arg-Gly-Asp; SNP, single nucleotide polymorphism.
Acute ischaemic coronary events continue to be the major cause of death in Type 2 diabetes [1]. Type 2 diabetes per se qualifies for the same risk as having experienced a first myocardial infarction $[2,3]$. The clinical ischaemia cascade of acute coronary syndromes is clearly a platelet-driven phenomenon [4]. Consequently, increased numbers of platelets circulate in an activated state in diabetic patients $[5,6]$. Increases in the constitutive number of platelet surface fibrinogen receptors (GPIIb-IIIa) led us to assume a primary "diabetic" thrombocytopathy which renders the peripheral platelet pool more susceptible towards activation [7]. 
Table 1. Clinical characteristics and biochemical data of Type 2 diabetes mellitus patients and non-diabetic controls

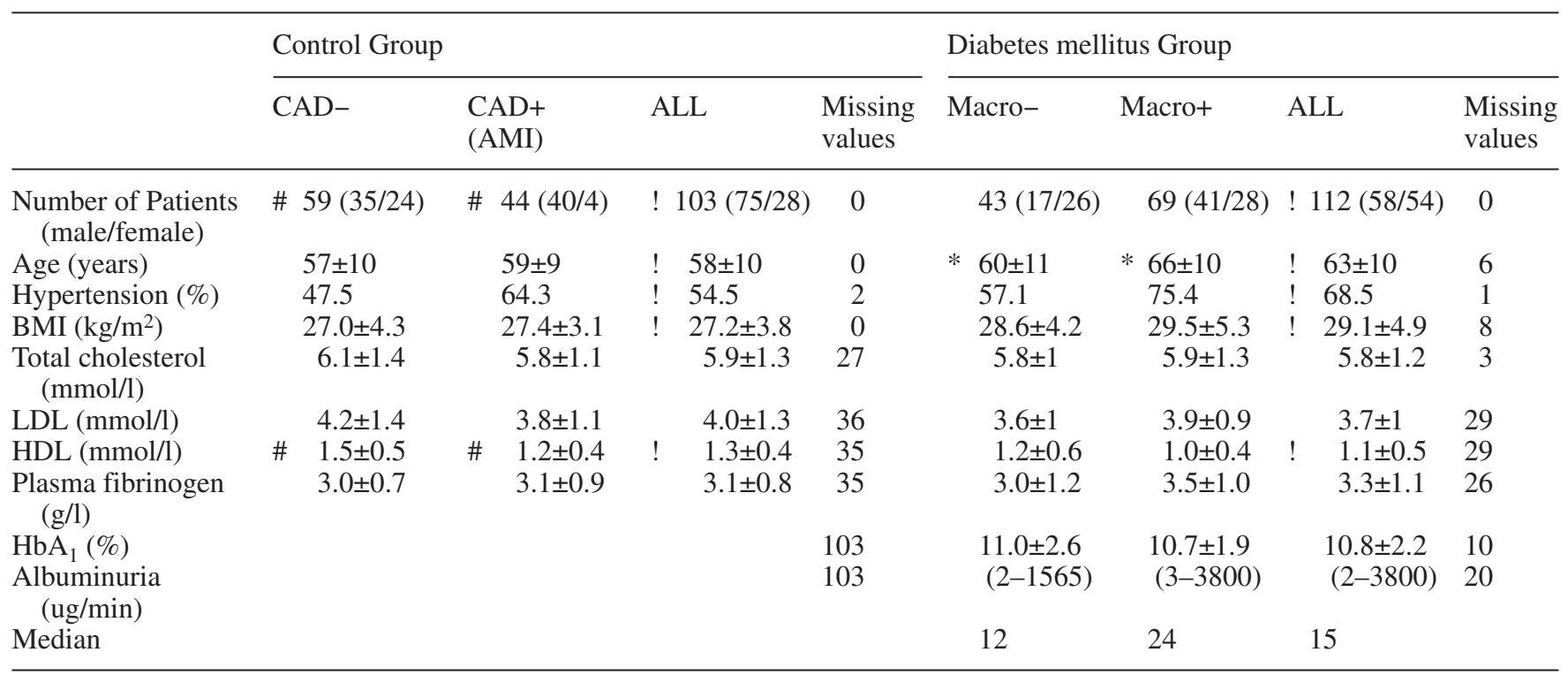

$\mathrm{AMI}=$ acute myocardial infarction; $\mathrm{BMI}=$ body mass index; $\mathrm{CAD}=$ coronary artery disease HDL $=$ high density lipoprotein; LDL = low density lipoprotein; Macro-/+ = without/with macrovascular disease
\#, * or ! p<0.05, two sided testing: \# CAD- vs. CAD+/AMI; * Macro- vs. Macro+; ! all controls vs. all diabetics
The nucleated bone marrow megakaryocytes have been shown to be altered both in terms of DNA content (ploidy) and quantitative GPIIb-IIIa expression in the diabetic BB-rat model and in human Type 2 diabetes patients $[8,9]$.

The platelet GPIIb-IIIa receptor converges the wide array of agonist-induced activation signalling towards functional platelet aggregation by fibrinogen crossbridging ("final common pathway"; [10]). The outside-in signalling cascade of this receptor is modulated by the proteolytic enzyme calpain, an isoform of which has shown a genetic polymorphism with tight association to the manifestation of clinical Type 2 diabetes mellitus [16).The gene encoding the $\beta_{3}$-subunit (GPIIIa) of the receptor which contains the RGD-recognizing sequence of the fibrinogen molecule, shows a polymorphism (PlA1/A1, PIA1/A2, PIA2/A2) suggested to promote more intense platelet- fibrinogenreceptor interaction [11, 12]. Clinically, others have reported for the first time the existence of a strong association between the genomic prevalence of the PlA2 allele and acute coronary thrombosis [13].

Along with the reported findings of DNA-changes in the nucleated megakaryocytic precursor cells under diabetic conditions, we found it intriguing to investigate whether genomic variation also exists in the GPIIb-IIIa gene in favour of the putative thrombogenic PlA2 allele and how the vascular status is related in diabetic patients as compared to a genetic control background.

\section{Subjects and methods}

Study design and patients. This investigation was designed as a cross-sectional study including consecutive patients with diabetes mellitus and non-diabetic control subjects. All investigations were carried out in accordance with the Declaration of Helsinki as revised in 2000.

Blood samples from a total of 215 subjects were analyzed. The diagnosis of diabetes required documented hyperglycaemia and increased $\mathrm{HbA}_{1}$. The type of diabetes was classified according to mode of treatment, anamnesis and clinical case presentation using recent American Diabetes Association guidelines [14]. In the diabetes group $(n=112)$, patients of the German Diabetes Research Institute, Duesseldorf with $(n=69)$ or without clinically apparent macrovascular complications $(n=43)$ were included. Macrovascular disease was assumed with the presence of symptoms (stenocardia, gangrene, amputation or confirmed apoplexia), angiologically proven atherosclerotic disease (peripheral, cerebral or coronary arteries), ischaemic ECG signs (ST-segment depression $\geq 1 \mathrm{mV}$ ) and the requirement of any targeting medication (e.g. nitrates). Endpoint reporting was based on recalling patient data, detailed review of hospital records using standard criteria and the recent clinical investigation.

The control group consisted of non-diabetic patients $(n=103)$ admitted to the cardiological department of the University Clinic Essen with unspecified chestpain where CAD was excluded $(n=59)$ or confirmed on the basis of coronary angiography and a proven history of AMI $(n=44)$ (Table 1).

PlA-genotyping. The genotype (PIA1/A1, PIA1/A2, PIA2/A2) was determined by restriction fragment length analysis (RFLA).

Genomic DNA was isolated from whole blood samples using the QIAamp Blood Kit (Qiagen, Hilden, Germany) according to the manufacturers protocol. The samples were stored at $-20^{\circ} \mathrm{C}$ in EDTA $(1.6 \mathrm{mg} / \mathrm{ml})$ coated collection tubes (Sarstedt, Nümbrecht, Germany). 
Exon two was amplified using the flanking primers $5^{\prime}$-TTC TGA TTG CTG GAC TTC TCT T-3'(sense)/ 5'TCT CTC CCC ATG GCA AAG AGT-3'(antisense). The PCR was done for 32 cycles $\left(92^{\circ} \mathrm{C} 30 \mathrm{~s} ; 55^{\circ} \mathrm{C} 1 \mathrm{~min} ; 72^{\circ} \mathrm{C} 1 \mathrm{~min}\right.$.) with a final extension at $72^{\circ} \mathrm{C}$ for 10 min (Hybraid-Omnigen-PCR, MWG-Biotech, Ebersberg, Germany). The amplification mix $(40 \mu \mathrm{l})$ contained $100 \mathrm{ng}$ of template DNA, $1.5 \mathrm{mmol} / \mathrm{l} \mathrm{MgCl}_{2}$, $200 \mu \mathrm{mol}$ of each dNTP, $2.5 \mathrm{pmol}$ of each primer, 1-times PCR-reaction buffer [20 mmol/1 Tris-HCl (pH 8,4), 50 mmol/1 $\mathrm{KCl}]$ and $25 \mathrm{mU} / \mu \mathrm{l}$ Taq-polymerase, both from Gibco-Life Technologies, (Eggenstein, Germany). The resulting PCR product (266 base-pairs) was further analysed by restriction analysis:

The mutation characterizing the PIA2-allele is a substitution of cytosine for thymidine at position 1565, AC $E \mathrm{BBL}_{\mathrm{J} 05427}$, in exon two of the platelet fibrinogen receptor on chromosome 17 [15]. Carrying this substitution the exon two PCR-amplificate (266 bp) can be restricted by Nci I $\left(5^{\prime}-\mathrm{CC}_{1565} \downarrow(\mathrm{C} / \mathrm{G}) \mathrm{GG}-3^{\prime}\right)$ creating two restriction fragments of 216 and 50 bp respectively and by Msp I (5'-C $\downarrow \mathbf{C}_{\mathbf{1 5 6 5}}$ GG-3') resulting in three restriction fragments of $171,50,45 \mathrm{bp}$ (enzymes from MBI-Fermentas, St. Leon-Rot, Germany). The wild-type allele PIAI is not cut by Nci I and only cut one time by Msp I giving two fragments of 216 and $50 \mathrm{bp}$. The restrictions were carried out overnight according to the manufacturers protocol. The resulting fragments were concentrated using $1 / 10$ volume sodium acetate $(3 \mathrm{~mol}, \mathrm{pH} 5.2)$ and 2.5 volume ethanol $(100 \%)$ for $12 \mathrm{~h}$ at $-20^{\circ} \mathrm{C}$. DNA was collected $\left(15,000 \mathrm{~g}, 4^{\circ} \mathrm{C}, 30 \mathrm{~min}\right)$ and redisolved in $5 \mu \mathrm{H}_{2} \mathrm{O}_{\text {autoclaved }}$ $3 \mu$ of loading buffer [5\% (v/v) glycerol, $0.04 \%$ (w/v) bromphenolblue and $0.04 \%(\mathrm{w} / \mathrm{v})$ xylenxyanol] were added and the restriction pattern was analysed in a $2 \%$ agarose gel (1×TAE) containing ethidium bromide $(0,5 \mu \mathrm{g} / \mathrm{ml})$.

SSCP-analysis and fluorescence sequencing of the PCRamplificates and digest products was done by spot checks and achieved complete confirmation.

Clinical chemistry. All documentation parameters ( $\mathrm{HbA}_{1}$, cholesterol, LDL-, HDL-cholesterol, fibrinogen) were determined using routine methods.

Statistical analysis. Categorial variables are shown by number or percentage. Continuous data are presented as means \pm SD or median and range. Categorial variables were compared by Fisher's exact test and continuous variables with Student's $t$-test or Wilcoxon-test.

Odds ratios (OR) are given with 95\% CI (MantelHaenszel). To adjust for confounders a multiple logistic regression model was carried out with dependent variable diabetes and covariates PIA-genotype, age, sex and BMI. Data were computed by SAS version 8.1 TS1M0 on UNIX. A $p$ value of less than 0.05 was considered statistically significant.

\section{Results}

Risk factor profile. There were slight, yet significant differences between the groups in the prevalence of hypertension, age and HDL cholesterol (Table 1). When comparing patient subgroups, fibrinogen was additionally increased in diabetic patients with macrovascular complications. There were no statistically definite associations of selected risk factors and PIA-genotype (data not shown).

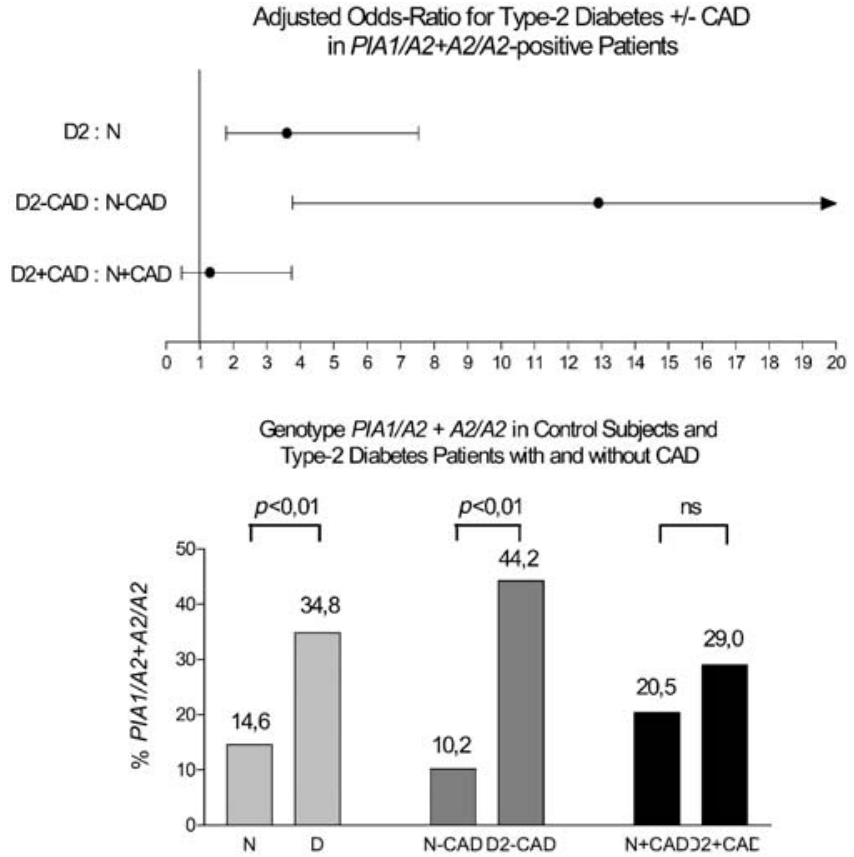

Fig. 1. (A) Odds ratios (adjusted) between PIA allele frequencies and clinical status. CAD $+/-=$ with/without coronary artery disease. (B) PIA2-prevalence by patient subgroups

Genotyping. The prevalence of the PlA2 allele (PlAX/A2: either heterozygous PlA1/A2 or homozygous PlA2/A2) among the diabetic patients accounts for $34.8 \%$ (39/112) being more than two-fold higher than among non-diabetic control subjects [14.6\%; 15/103; OR 3.1 (1.6-6.1), $p<0.01$ ] (Fig. 1A,B).

Subgroup comparison of Type 2 diabetes patients without vascular complications with non-diabetic control subjects without documented coronary artery disease resulted in an even greater separation of PlA2allele-positivity: $44.2 \%(19 / 43)$ compared with $10.2 \%$ (6/59); OR 7.0 (2.5-19.7), $p<0.01$.

Multivariate analysis. The magnitude of the reported odds ratios did only slightly change after multivariate adjustment for sex, age and BMI (data set complete with 10 dropouts because of missing values): OR 3.6 (1.7-7.5), and OR 12.9 (3.8-43.2), $(p<0.01, p<0.01)$ (Fig. 1A).

Vascular disease. In contrast, the separation was lost when exclusively comparing the Type 2 diabetic patients with vascular disease with the non-diabetic control subjects with documented coronary artery disease: $29.0 \%(20 / 69)$ compared with 20.5\% (9/44); OR 1.6 (0.6-3.9), [OR adjusted: $1.3(0.5-3.8)$ ].

CAD/AMI patients without diabetes show a twofold higher PlA2 carriage $(20.5 \%$; 9/44) than subjects without CAD $(10.2 \% ; 6 / 59)$. In the Type 2 diabetes group there was no difference in the prevalence of PlA2 whether vascular complications were detectable (29.0\%; 20/69) or not $(44,2 \%, 19 / 43)$ (Fig. 1B). 


\section{Discussion}

We report a higher prevalence of the PIA2-allele of the gene encoding for the GPIIIa $\left(\beta_{3}\right)$ subunit of the platelet surface $\alpha_{2} \beta_{3}$ - integrin (GPIIb-IIIa) in patients with Type 2 diabetes mellitus as compared to non-diabetic control subjects. The association of this single nucleotide polymorphism (SNP) was strong with the metabolic disease, but absent with the manifestation of clinical atherosclerotic disease.

This represents an unexpected new finding for Type 2 diabetes patients being at a particular high risk for the development of coronary artery disease. Our cross-sectional linkage analysis with clinical phenomenology clearly provides no causative relationship. However, similar to the association of genetic variations in the calpain-10 gene our finding here suggests that platelet pathways not yet considered may be of some pathogenetic impact for the metabolic disease or inherently belong to the wide array of pathophysiologic alterations linked to Type 2 diabetes [16]. Agonistdependent calpain activation is recognized as part of the outside-in stimulus response pathways being implicated in the procoagulant activity of activated platelets [17]. Recently, this enzyme has been shown to proteolyse structural and signalling proteins involved in cytoskeleton remodelling and platelet activation events downstream to $\alpha_{2} \beta_{3}$ - integrin (GPIIb-IIIa) activation $[18,19]$. Unfortunately, we do not have parallel genomic calpain data available in this limited cohort study, but our results of a tight genetic association of the $\alpha_{2} \beta_{3}$ - integrin (GPIIb-IIIa) physiology (not exclusively restricted to platelets) with Type 2 diabetes could provide an additional pathway as to how genetic variations in the calpain system might functionally operate. Moreover, an interaction of both genetic variations could be anticipated, but the nature of this remains completely speculative.

We cannot provide genomic linkage data of the investigated SNP with other genetic variations in close proximity of the PlA2 gene locus (17q21.32). GLUT-4 and the glucagon-receptor are known Type 2 diabetes related genes, but both are located at the opposite end of the chromosome $(17 \mathrm{p} 13 ; 17 \mathrm{q} 25)$ suggesting no interaction with the SNP under investigation.

Based on antibody binding data it is assumed that the PIA2- genotype is processed into functionally relevant phenotypical alterations of ligand (fibrinogen)receptor (GPIIb-IIIa) interaction possibly causing hyperaggregation in response to stimulating agonists such as shear, matrix components or coagulation inducers [20, 21, 22, 23, 24]. Clearly, PIA2- carriers generate more thrombin after microvascular trauma which underlines the functional meaning of this particular platelet SNP also suggesting some pharmagenomic importance $[25,26]$. A more complex interpretation can be derived from the data from another study showing that ligation of the PIA2-genotype integrin could affect outside-in signalling efficacy with the consequence of more intense platelet-platelet interaction, but also increased adherence to immobilized adhesion molecules. Such events might take place not only in the large high flow vessels, but also in the nutritive microcirculation being regulatory part of the skeletal muscle energy metabolism [27, 28].

Our data further promulgate the earlier suggestion of a primary, genetically-determined link between diabetes and platelet physiology which could operate via the GPIIb-IIIa platelet surface integrin. This receptor was phenotypically shown to be increased on platelets from diabetic patients, but in the BB-rat model of insulin deficient diabetes increased GPIIb-IIIa-expression was also accompanied by changes in DNA-content (ploidy) of the nucleated megakaryocytic bone marrow precursor cells [9]. It was therefore consequent to ask for genetic prothrombotic variants associated with platelet hyper-reactivity in Type 2 diabetic patients. The unequivocally clear answer from our data shows that the PIA2-positive genotype is closely associated with the diagnosis of diabetes suggesting a possible relationship with the metabolic disease itself. It is tempting to speculate therefore, that the PIA-SNP belongs to the diabetes-related genetic variation which also operates lineage-specific blood cell lines [29, 30]. The PIA2-type megakaryocyte-platelet axis could then result in a highly reactive peripheral platelet pool which responds to lower activation thresholds ("activated megakaryocyte-platelet-system" [7]).

In non-diabetic patients our data support the assumption of a possible association with the risk of acute ischaemic coronary syndromes trendwise by the observed doubling in the allelic frequency: 10.2 compared with $20.5 \%$ (non significant). Increased PIA2prevalence in CAD and in patients after myocardial infarction was reported $[31,32,33,34,35]$. In a prospective nested case control trial within the Physician's Health Study no association was found of the PIA2-prevalence with any thrombotic endpoint at all and this was confirmed by the ECTIM and ARIC studies and by some smaller studies [36, 37, 38, 39, 40]. Others seem to resolve these striking differences by their suggestion that PIA2-prevalence does not act as a risk factor per se, but strongly determines the thrombogenic reactivity of the circulating platelets [32]. This concept is attractive, since it addresses platelet hyperreactivity super-imposed to coronary atherosclerosis to act as the final mechanism which translates risk factor-dependent vessel wall morphology into blood-dependent thrombotic infarction. It is interesting to note that no interference must be anticipated with the newly-recognized myocardial infarction susceptibility gene on chromosome 14 [41]. However, a relation between PIA2-prevalence and cholesterol levels as well as with smoking upon ischaemic endpoints was reported clearly suggesting an interaction between the genotype and environmental factors 
$[42,43]$. In turn, prevalence of diabetes could have contributed skewness to the clinical and epidemiological studies mentioned above thus accounting for some of the contradictory results.

The magnitude of our observation might depend on the unexpected low PIA2- prevalence in the non-diabetic control subjects which potentially reflects a high selection bias with this special population of chest pain patients. However, the increase of the PIA2prevalence above $30 \%$, exceeding the ranges observed with $\mathrm{CAD}$, and a history of myocardial infarction in simple association with the presence of Type 2 diabetes fits with earlier observations. One study found a PIA2- prevalence up to $38 \%$ in Type 2 diabetic patients with macrovascular disease before the age of 60 being not significantly different from $29 \%$ in patients without macrovascular disease [43].

In summary, our cross-sectional analysis suggests a close association of the PIA2 SNP with Type 2 diabetes mellitus. The nature of this association cannot be answered by this limited data set, but the view that a prothrombotic genetic variation associates with the metabolic disease further supports the "common soil" hypothesis: that the presence of the PIA2 allele could precipitate thrombotic endpoints, explaining in part the extraordinary risk of these patients for acute ischaemic events. Thus, we propose PIA2 SNP as a diabetes related genetic variation being secondarily involved in the macrovascular prognosis of such patients. However, this hypothesis urgently awaits confirmation from the prospective epidemiological KORA trial data.

Acknowledgements. We thank Mrs. H. Liu and Dr. K. Stockklauser-Färber for their excellent assistance in carrying out the molecular genetic techniques and critical methods review. This work was in part supported by a grant from the German Diabetes Association.

\section{References}

1. Nathan DM, Meigs J, Singer E (1997) The epidemiology of cardiovascular disease in type 2 diabetes mellitus: how sweet it is... or is it? Lancet 350 [Suppl I]:4-9

2. Haffner S, Lehto S, Rönnemaa T, Pyorälä K, Laakso M (1998) Mortality from coronary heart disease in subjects with type-2 diabetes and in nondiabetic subjects with and without prior myocardial infarction. New Engl J Med 339:229-234

3. Tschoepe D (1998) Coronary artery disease in diabetes mellitus: a challenge for intervention. Exp Clin Endocrinol Diabetes 106:16-24

4. Topol EJ (1998) Toward: new frontier in myocardial reperfusion therapy. Emerging platelet preeminence. Circulation 97:211-218

5. Tschoepe D, Driesch E, Schwippert B, Niewenhuis HK, Gries FA (1995) Exposure of adhesion molecules on activated platelets in patients with newly diagnosed IDDM is not normalized by near- normoglycemia. Diabetes 44:890895
6. Tschoepe D, Roesen P, Esser J et al. (1990) Large platelets circulate in an activated state in diabetes mellitus. Semin Thromb Hemost 17:433-439

7. Tschoepe D (1995) The activated megakaryocyte- platelet system in vascular disease: focus on diabetes. Semin Thromb Hemost 21:152-159

8. Brown A, Hong Y, Belder A de, Beeso HL, Sherword R (1997) Megakaryocyte ploidy and platelet changes in human diabetes and atherosclerosis. Arterioscl Thromb Vasc Biol 17:802-807

9. Tschoepe D, Schwippert B, Schettler B, Kiesel U, Rothe H, Roesen P (1992) Increased GPIIBIIIA expression and altered DNA-ploidy pattern in megakaryocytes of diabetic BB-rats. Eur J Clin Invest 22:591-598

10. Levkovits J, Plow EF, Topol EJ (1995) Platelet glycoprotein IIb/IIIa receptors in cardiovascular medicine. New Engl J Med 332:1553-1559

11. Zimrin AB, Gidwitz S, Lord S et al. (1990) The genomic organization of platelet glycoprotein IIIa. J Biol Chem 265:8590-8595

12. Kunicki TJ, Newman PJ (1992) The molecular immunology of human platelet proteins. Blood 80:1386-1404

13. Weiss EJ, Bray PF, Tayback M et al. (1996) A polymorphism of a platelet glycoprotein receptor as an inherited risk factor for coronary thrombosis. New Engl J Med 334:1090-1094

14. The Expert Committee on the Diagnosis and Classification of Diabetes Mellitus (1997) Report of the Expert Committee on the Diagnosis and Classification of Diabetes Mellitus. Diabetes Care 20:1183-1193

15. Newman PJ, Derbes RS, Aster RH (1989) The human platelet alloantigens. PIA1 and PIA2 are associated with a leucine 33/proline 33 amino acid polymorphism in membrane glycoprotein IIIa, and are distinguishable by DNA typing. J Clin Invest 83:1778-1781

16. Horikawa Y, Oda N, Cox NJ et al. (2000) Genetic variation in the gene encoding calpain-10 is associated with type 2 diabetes mellitus. Nat Genet 26:163-175

17. Fox JE, Reynolds CC, Austin CD (1990) The role of calpain in stimulus-response coupling: evidence that calpain mediates agonist-induced expression of procoagulant activity in platelets. Blood 76:2510-2519

18. Croce K, Flaumenhaft R, Rivers M et al. (1999) Inhibition of calpain blocks platelet secretion, aggregation, and spreading. J Biol Chem 274:36321-36327

19. Schoenwaelder SM, Yuan Y, Jackson SP (2000) Calpain regulation of integrin alpha IIb beta 3 signaling in human platelets. Platelets 11:189-198

20. Weiss EJ, Goldschmidt-Clermont PJ, Grigoryev D, Jin Y, Kickler TS, Bray PF (1995) A monoclonal antibody (SZ21) specific for platelet GPIIIa distinguishes PIA1 from PIA2. Tissue Antigens 46:374-381

21. Schwippert-Houtermans B, Strapatsakis S, Roesen P, Tschoepe D (2001) Evaluation of an antibody-based genotype classification of the platelet fibrinogen receptor (GPIIbIIIa). Comm Clin Cytometry 46:238-242

22. Feng D, Lindpainter K, Larson MG et al. (2001) Platelet glycoprotein IIIa PIA2 polymorphism, fibrinogen and platelet aggregability. The Framingham Heart Study. Circulation 104:140-144

23. Cannon CP, Bray PF, Ault K, Rizzo MJ, Braunwald E (1998) PIA2 polymorphism of the platelet IIbIIIa receptor: increased risk of early recurrent ischemic events in acute coronary syndromes, increased platelet aggregability, and effective platelet inhibition by an oral IIbIIIa inhibitor: Results from TIMI 12. Circulation 98 [Suppl 1]:A880 
24. Ripoll L, Kereveur A, Mazoyer E et al. (1998) PIA platelet GPIIbIIIa polymorphism is associated with increased platelet aggregation and risk of ischemic stroke in the young. Circulation 98 [Suppl 1]:A2409

25. Undas A, Brummel K, Musial J, Mann K, Szczeklik A (2001) PIA2 polymorphism of $\beta 3$ integrins is associated with enhanced thrombin generation and impaired antithrombotic action of aspirin at the site of microvascular injury. Circulation 104:2666-2672

26. Michelson AD, Furman MJ, Goldschmidt-Clermont $\mathrm{P}$ et al. (2000) Platelet GPIIIa PIA polymorphisms display different sensitivities to agonists. Circulation 101:1013-1018

27. Vijayan KV, Goldschmidt-Clermont PJ, Roos C, Bray PF (2000) The PIA2 polymorphism of integrin $\beta 3$ enhances outside-in signaling and adhesive functions. J Clin Invest 105:793-802

28. Scherrer U, Sartori C (2000) Defective nitric oxide synthesis: a link between metabolic insulin resistance, sympathetic overactivity and cardiovascular morbidity. Eur J Endocrinol 142:315-323

29. Lampeter E, Homber M, Quabeck K et al. (1993) Transfer of insulin-dependent diabetes between HLA-identical siblings by bone marrow transplantation. Lancet 341:1243-1244

30. Kim HO, Blakemore K, Kwon OH et al. (1995) Gene frequencies of the five major human platelet antigens in African American, white, and Korean populations. Transfusion 35:863-867

31. Gardemann A, Humme J, Stricker J et al. (1998) Association of the platelet glycoprotein IIIa PIA1/A2 gene polymorphism to coronary artery disease but not to nonfatal myocardial infarction in low risk patients. Thromb Haemost 80:214-217

32. Zotz RB, Winkelmann BR, Nauck M et al. (1998) Polymorphism of platelet membrane glycoprotein IIIa: human platelet antigen 1b (HPA-1b/PIA2) is an inherited risk factor for premature myocardial infarction in coronary artery disease. Thromb Haemost 79:731-735

33. Garcia-Ribes M, Gonzales-Lamuno D, HernandezEstefania R et al. (1998) Polymorphism of the platelet glycoprotein IIIa gene in patients with coronary stenosis. Thromb Haemost 79:1126-1129
34. Carter AM, Ossei-Gerning N, Wilson IJ, Grant PJ (1997) Association of the platelet PlA polymorphism of glycoprotein IIb/IIIa and the fibrinogen B $\beta 448$ polymorphism with myocardial infarction and extent of coronary artery disease. Circulation 96:1424-1431

35. Walter DH, Schächinger V, Elsner M, Dimmeler S, Zeiher AM (1997) Platelet glycoprotein IIIa polymorphisms and risk of coronary stent thrombosis. Lancet 350:12171219

36. Ridker PM, Hennekens CH, Schmitz C, Stampfer MJ, Lindpaintner K (1997) PlA1/A2 polymorphism of platelet glycoprotein IIIa and risks of myocardial infarction, stroke, and venous thrombosis. Lancet 349:385-388

37. Herrmann SM, Porier O, Marques-Vidal P et al. (1997) The Leu 33/Pro polymorphism (PlA1/A2) of the glycoprotein IIIa (GPIIIa) receptor is not related to myocardial infarction in the ECTIM study. Thromb Haemost 77:11791181

38. Aleksic N, Nuneja H, Folsom AR et al. (2000) Platelet PIA2 allele and incidence of coronary heart disease. Results from the Atherosclerosis Risk in Communities (ARIC) Study. Circulation 102:1901-1905

39. Scaglione L, Bergerones S, Gaschino G et al. (1998) Lack of relationship between the PlA1/PlA2 polymorphism of platelet glycoprotein IIIa and premature myocardial infarction. Eur J Clin Invest 28:385-388

40. Durante-Mangoni E, Davies GJ, Ahmed N, Ruggiero G, Tuddenham EG (1998) Coronary thrombosis and the platelet glycoprotein IIIA gene PLA2 polymorphism. Thromb Haemost 80:218-219

41. Broeckel U, Hengstenberg C, Mayer B et al. (2002) A comprehensive linkage analysis for myocardial infarction and its related risk factors. Nat Genet 30:210-214

42. Merlini PA, Niguarda O, Ardissimo D et al. (1998) Interactions between environmental and prothrombotic genetic risk factors in young survivors of myocardial infarction. Circulation 98 [Suppl 1]:A2408

43. Carter AM, Mansfield MW, Grant PJ (1998) Polymorphisms of platelet glycoproteins in relation to macrovascular disease in type 2 diabetes mellitus. Diabet Med $15: 315-319$ 